\title{
Research of Data Fusion Method of High Power Transient Electric Field Signal
}

\author{
Xiang Chen ${ }^{1, \text { a }}$, Yangyang Wang ${ }^{2, \mathrm{~b}}$, Zheng $\mathrm{Li}^{2}$ \\ ${ }^{1}$ State Key Laboratory of Complex Electromagnetic Effects on Electronics Information System, \\ Luoyang, 471023, China \\ ${ }^{2}$ Department of Photoelectric and Space, Electronic Engineering Institute of PLA, Hefei, 230037, \\ China \\ aemail: chenxiang061101@163.com, bemail: 460974520@qq.com
}

Keywords: High Power Transient Electric Field; Sensor; Data Fusion

\begin{abstract}
High power transient electric field is coupled into the interior of the electronic system easily. How to detect the field signal and reduce the influence of the field has become one of the most important fundamentality research projects. However, single sensor cannot detect the wide bandwidth signal effectively. According to the problem that the detecting sensor cannot cover the bandwidth of the signal, this paper presented a method of data fusion to combine the signal of different sensors to restore the original high power transient electric field signal. Experiments show that the method features a quick response and high accuracy of the restoration of the original high power transient electric field signal generated by the experimental system.
\end{abstract}

\section{Introduction}

The high power transient electric field typically has nanosecond or sub nanosecond rise time and extremely high field strength, which leads the damage to the electronic equipment easily. The high power transient electric field usually has highly rapid rising edge, very short duration time and very high peak value in the time domain, while in the frequency domain, it can cover wide frequency band, and therefore a strict requirement of the measurement instrument is needed[1].

High power transient electric field is coupled into the interior of the electronic system easily because of its wide bandwidth and big field strength. With the development of the technology, the influence of fields such as military, national defense and communication caused by high power electromagnetic environment will become bigger and bigger. In recent years, the research of electronic-magnetic effect and its protection strategy of the high power transient electric field had been one of the most important fundamentality research projects[2,3].

According to the wide bandwidth of high power transient electric field, single sensor cannot detect it effectively. Two or more sensors were applied in this paper and a data fusion method was applied in the restoration of the high power transient electric field. The experimental system constructed in the paper showed the quick response and high accuracy of the method.

\section{Data Fusion Technology}

The data fusion technology is a signal processing technology developing rapidly in the field of information science. Generally speaking, data fusion is the process of various field such as detection, relevancy, estimation and comprehension of the redundant or complementary information and data of many sources. During the process of fusing, all the useful features of all the signal will be preserved and the optimum unify estimation of the measured object will be obtained.

There are many advantages of using multi-sensors to extract and restore the high power transient electric field signal, such as the reduction of the sensibility of the environmental noise counter to the time variation and the best use of complementary characteristics of multi sensors data, to make up the defection that the data structure from single sensor is too simple.

In the process of data fusion with multi sensors, the sensors can be the same type or the different 
type. Therefore the measurement result of the same object of different sensors will different to some extent. A judgement should be applied to the data detected by the sensors to decide whether the data is credible. It is an effective method to define the matrix of relation by statistical methods, to measure the correlation degree of the sensors.

Supposed there are several sensors to measure the same signal, the measurement value should satisfy the Gaussian distribution. If the confidence distance measure $d_{i j}$ is introduced to express the deviation of two sensors, the smaller of the confidence distance measure, and the closer of the measurement values. If the sensors are the same, $d_{i j}$ equals 0 . All the confidence distance measure makes up the distance matrix. When the confidence distance measure is smaller than threshold $\varepsilon$, the two sensors can be considered as mutual supported with the value is 1 , otherwise the value is 0 . Then the matrix of relation is constituted as formula 1[4].

$$
R_{n}=\left[\begin{array}{cccc}
r_{11} & r_{12} & \cdots & r_{1 n} \\
r_{21} & r_{22} & \cdots & r_{2 n} \\
\cdots & \cdots & \cdots & \cdots \\
r_{n 1} & r_{n 2} & \cdots & r_{n n}
\end{array}\right]
$$

In formula $1, r_{i j}$ means the supporting degree of the No.i sensor from No. $j$ sensor. The threshold $\varepsilon$ is usually selected by experience, which will affect the final fusion result.

Bayes norm was applied in the data fusion algorithm. Supposed there were $\mathrm{n}$ sensors that detected the same high power transient electric signal. The optimum fusion number is $l(l \leq n)$ and the fusion set $X=\left(x_{1}, x_{2}, \ldots, x_{l}\right)$. The conditional probability density of each measurement value was set as formula 2.

$$
\mathrm{P}=\left(\mu \mid x_{1}, x_{2}, \ldots, x_{l}\right)=\frac{P\left(\mu ; x_{1}, x_{2}, \ldots, x_{l}\right)}{P\left(x_{1}, x_{2}, \ldots, x_{l}\right)}
$$

In formula 2, the $\mu$ was the mean value of the measurement. The parameter $\mu$ complied with Gaussian distribution $N\left(\mu_{0}, \sigma_{0}^{2}\right)$, and $x_{k}$ complied with $N\left(\mu, \sigma_{k}^{2}\right)$. Therefore, the Bayes estimation of $\mu$ was set as formula 3 .

$$
\hat{\mu}=\int \mu \frac{1}{\sqrt{2} \pi \sigma_{\mathrm{N}}} \exp \left[-\frac{1}{2}\left(\frac{\mu-\mu_{N}}{\sigma_{N}}\right)^{2}\right] d \mu
$$

The high power transient electric field signal could be restored by the fusion of several sensors in different frequency bandwidth[5]. The data fusion algorithm could be divided into five steps that was shown as followed.

Step 1: the initialization of the original data. There are $n$ sensors in the measurement of the original signal. Normalization will be carried out in the data of the sensors.

Step 2: calculating the different value between the measurement value and the reference value. The different value can be considered as $\Delta \varphi_{n}$.

Step 3: according to formula 4, the amplitude of the detecting signal of the sensor in frequency $f$ can be calculated. In formula $4, p$ is the adjusting constant, which is related to the characteristics of the sensor, the circuit, and so on.

$$
V_{n, f}=p \cdot \Delta \varphi_{n, f}
$$

Step 4: the value of the sampling site of the original signal can be estimated through formula 3.

Step 5: after estimating all the value from the $n$ sensors, the whole value of each sampling site in time domain can be calculated and the restoring data can be acquired. 


\section{Experiment Results}

The structure diagram of the testing equipment of the high power transient electric field was designed as figure 1. This equipment was consisted by sensor, optical fiber and light receiver. During the testing time, the sensor was set in the high power transient electric field. The electric field signal was modulated to light signal by the active electric-light conversion circuit. After been transmitted to the optical receiver, the light signal could be modulated to electric signal during the optical-electrical conversion circuit, which could be recorded by the scope.

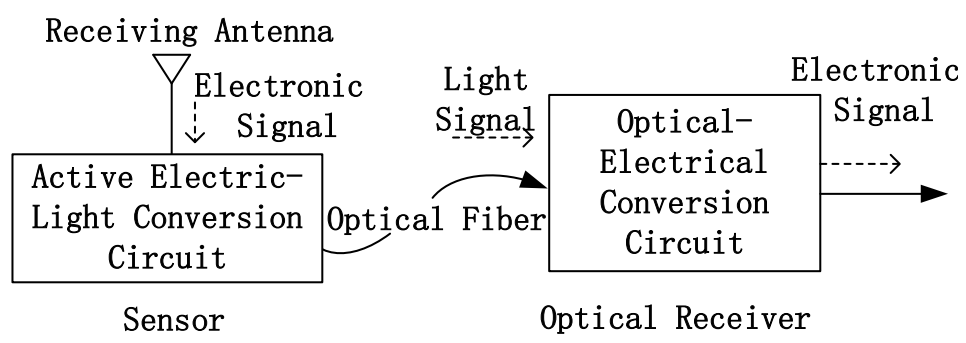

Figure 1 . The structure diagram of the testing equipment

The experimental environment was set as Figure 2. The pulse noise simulator was selected as NOISEKEN INS-4040 and the Scope was selected as Tektronix TDS7404B. The capacity of the antenna load was $2.4 \mathrm{nF}$, the amplitude of the input square wave was $100 \mathrm{~V}$, and the distance between the sensor and the board was $0.5 \mathrm{~m}$.

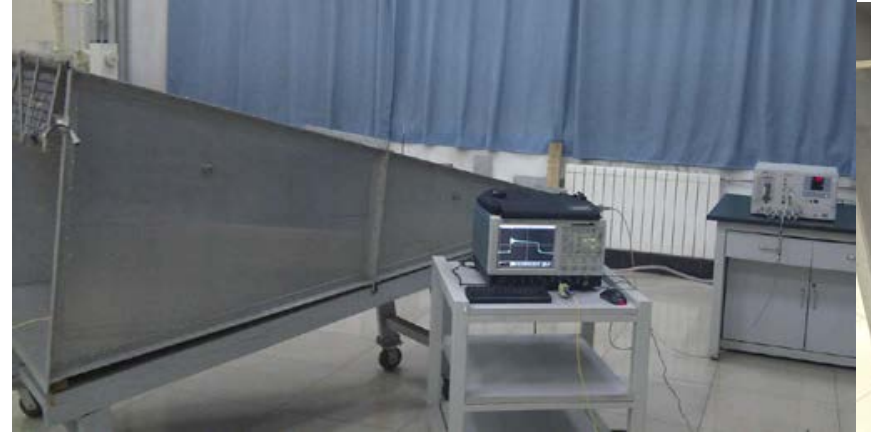

(a) the testing environment

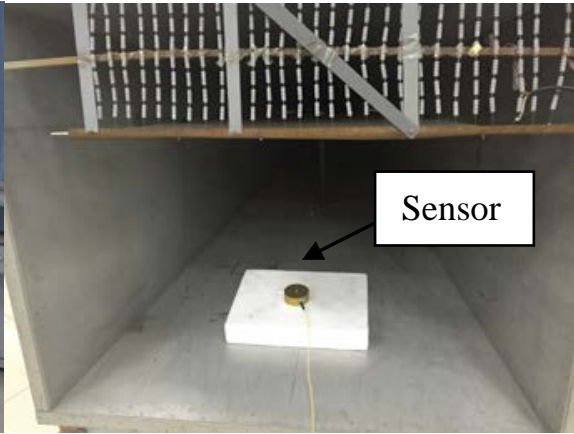

(b) the position of the sensor

Figure 2. The structure diagram of the testing equipment

The signal caught by the sensor was shown in Figure 3.

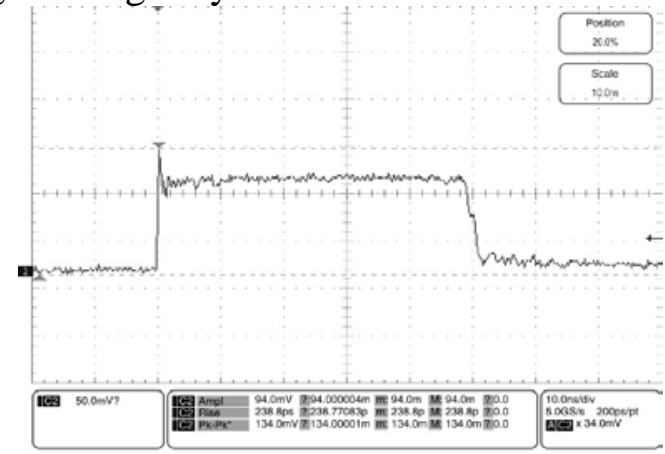

(a)The input square wave

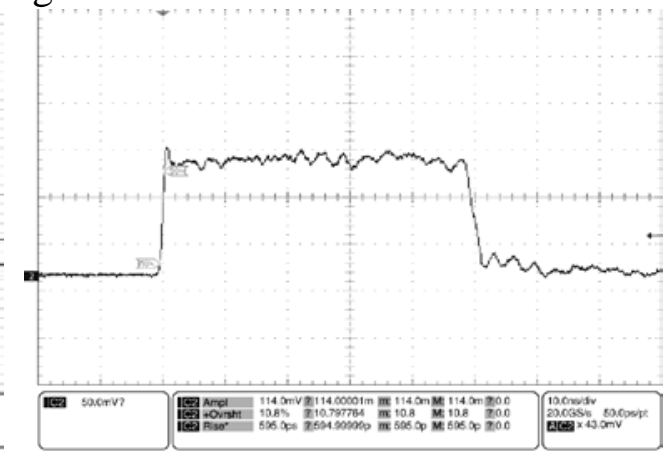

(b) the measuring square wave

Figure 3. The input and measured waveform with pulsed width of 50ns and amplitude of 100V

The pulse width of the square wave pulse generated by the noise simulator was set as 50ns and the amplitude of the pulse was set as $100 \mathrm{~V}$, which was shown as figure 3 . From the figure, the rising edge of the square wave was $238.8 \mathrm{ps}$ with the amplitude was $94 \mathrm{mV}$. The rising edge of the measurement wave from the sensor was 595ps, with the amplitude was $114 \mathrm{mV}$. That meant there was some broaden of the rising edge between the input wave and the measurement wave, but the sensor could detect the square wave pulse whose rising edge was less than 1ns, which could verify the good high frequency performance of the sensor. The gain of the experimental instrument was about $1.5 \mathrm{~dB}$ from calculation, which was in the $3 \mathrm{~dB}$ range of the sweep frequency analysis. From the formula 5, the upper cutoff frequency was about $588 \mathrm{MHz}$. 


$$
f_{H}=0.35 / t_{r}
$$

There were four sensors using in the experiment and because the probe had changed to $\times 10$ level, the value of the signal was not so big. The restoration of the signal calculated by Matlab was shown in Figure 4.

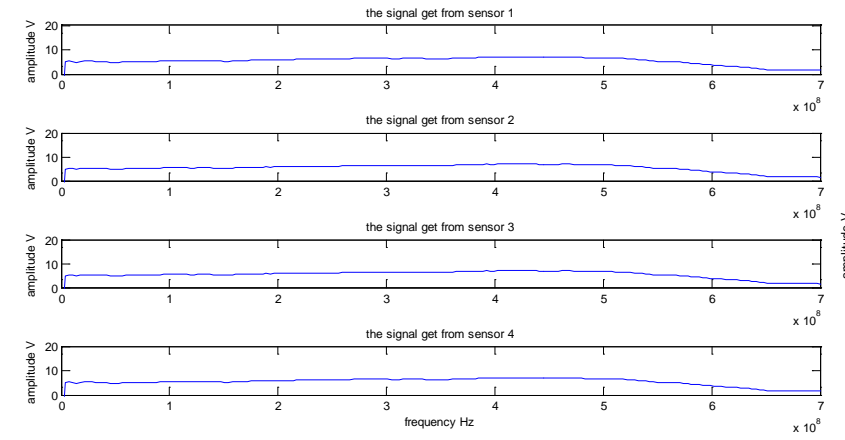

(a) The signal get from four different signal

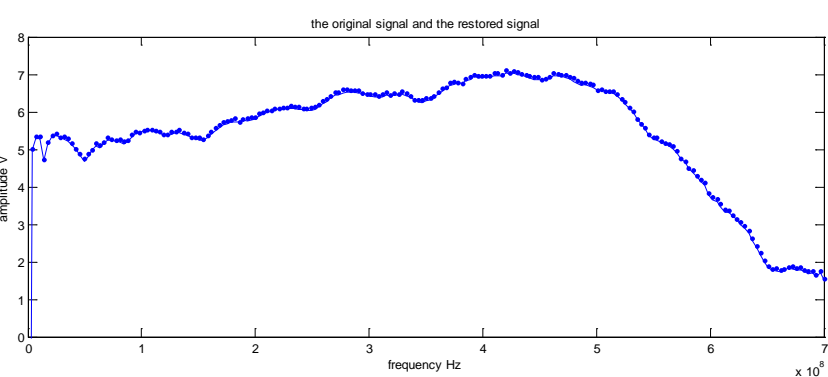

(b) the original and restored signal

Figure 4. The signal get from different signal and the original and restored signal

The signals caught from the four different sensors were shown in figure 4(a). Under the same experimental environment, the difference between the signals from the four sensors were not very great. The figure 4(b) showed the original signal and the restored signal after applying the algorithm, that the line meant original signal and the dotted line meant the restored signal. The restored signal matched the line of the original signal fairly well, which rectified the accuracy of the data fusion algorithm.

\section{Conclusion}

In the paper, a method of data fusion was introduced to detect the high power transient electric field. The experimental system was constructed and some experiments were carried out. Four sensors were applied in this paper and the experiment showed that this method had quick response and high accuracy in the detection.

\section{Acknowledgement}

In this paper, the research was sponsored by the Open Foundation of State Key Laboratory of Complex Electromagnetic Effects on Electronics Information System(Project No. CEMEE2015K0103B).

\section{References}

[1] Liu Shanghe. Effect of Electromagnetic Environment to Weaponry and Its Trend of Development [J]. Journal of the Academy of Equipment Command \& Technology. 2005,16(1):1-6

[2] F. Krng, P. Russer. The time-domain electromagnetic interference measurement system [J]. IEEE Trans. On EMC, 2003(3):330-338.

[3] Huang Ying, Tao Yungang, Zhou Jiemin, Su Dengjun. Implementation of D-S Evidential Theory in Multisensor Data Fusion System [J]. Journal of Nanjing University of Aeronautics \& Astronautics. 1999(2):16-23

[4] Wang Wei, Zhou Junhong, Wang Runsheng. A method of the multisensory data fusion [J]. Journal of Transducer Technology. 2003,22(9):39-41

[5] Shen Bin, Li Jun, Chen Xinqin. Data Fusion in Signals of Electric Fields of a Ship [J]. Ship Elctronic Engineering. 2009,29(5):169-171 